\title{
A New Method for Comprehensive Evaluation of Air Quality in Urban Agglomeration
}

\author{
Li Lei ${ }^{*}$, Xinli Chen², Wei Liu ${ }^{3}$ \\ ${ }^{1}$ Business School, Jiangnan University, Wuxi, China \\ ${ }^{2}$ School of Social Development and Public Policy, Fudan University, Shanghai, China \\ ${ }^{3}$ School of Internet of Things Engineering, Jiangnan University, Wuxi, China \\ Email: *lilei59@jiangnan.edu.cn
}

How to cite this paper: Lei, L., Chen, X.L. and Liu, W. (2021) A New Method for Comprehensive Evaluation of Air Quality in Urban Agglomeration. Atmospheric and Climate Sciences, 11, 547-562.

https://doi.org/10.4236/acs.2021.113033

Received: June 15, 2021

Accepted: July 4, 2021

Published: July 7, 2021

Copyright $\odot 2021$ by author(s) and Scientific Research Publishing Inc. This work is licensed under the Creative Commons Attribution International License (CC BY 4.0).

http://creativecommons.org/licenses/by/4.0/

\begin{abstract}
In this study, a new method for a comprehensive evaluation of air quality in urban agglomerations was developed based on a prototype used to solve the spatial Steiner-Weber point. With this method, the air quality information of each city in the city group is aggregated into an optimal gathering point, and then the air quality of the city group is then dynamically evaluated each year. According to the relevant data of the China Statistical Yearbook 2018, we applied this method to aggregate the air quality indices of the major cities in the Beijing-Tianjin-Hebei urban agglomeration from 2014 to 2017. Using the plant growth simulation algorithm (PGSA), the optimal assembly points were calculated to be of a higher accuracy, compared to the traditional mean value aggregation method. Finally, the air quality of the Beijing-Tianjin-Hebei urban agglomeration during each year was evaluated dynamically based on the obtained assembly points. The results show that the air quality of the urban agglomeration is ranked as follows: Y2016 $\succ$ Y2015 $\succ$ Y2017 $\succ$ Y 2014 .
\end{abstract}

\section{Keywords}

Urban Agglomeration, Air Quality, Optimal Information Aggregation, Dynamical Evaluation, Plant Growth Simulation Algorithm

\section{Introduction}

As cities become more modernized, international, and larger in scale, urban agglomeration becomes an inevitable trend of city development. However, the high concentration of populations and industries in urban agglomerations has also brought about serious environmental problems. Compared to the countryside or 
smaller-scale cities, contaminants in urban agglomerations often have greater adverse effects on the environment owing to such effects as accumulation, migration, and diffusion [1] [2]. At present, air pollutants in China mainly come from cities, particularly large cities with a permanent population of over 1 million. An urban agglomeration consisting of many cities can even cause serious air pollution. In order to deal with the problem, scholars had developed various evaluation methods for comprehensively evaluating the air pollution status of cities and regions. In 2008, more than 200 scientists from 35 countries participated in the International Symposium on Air Quality Management. At the meeting, an air quality assessment, the selection of practice, and evaluation criteria in urban and regional areas were extensively discussed [3], which has had a far-reaching impact.

In recent years, some scholars have chosen urban agglomerations with a large economic scale and high population density as the target research areas, namely, the Beijing-Tianjin-Hebei agglomeration, Yangtze River Delta, and Pearl River Delta region, and conducted empirical research on a comprehensive evaluation of the air quality in the region based on air quality monitoring data, which has yielded valuable research results [4]-[9]. More scholars focused on multi-angle and multi-disciplinary research into the theory and methods of air quality assessment in cities or urban agglomerations. For instance, Coelho M.C. built a regional air quality modeling system and used a combination of top-down and bottom-up methods to establish an emission inventory [10]. Wang N. et al. conducted simulation studies of emissions and energy consumption using the Weather Research and Forecasting-Community Multi-scale Air Quality (WRFCMAQ) model, which was based on air quality statistics (including atmospheric observation data) of the South China Pearl River Delta (PRD) for 2006-2014 [11]. Thunis P. et al. proposed a new method for a comprehensive assessment of air quality focused on regional and urban aspects. It was found that an evaluation model can assess the contribution of different regions or any specific location to the overall air pollution of the regions or cities [12]. The author also established a structured online database, and derived evaluation results combining the current comprehensive evaluation modeling methods with those of other scholars [13]. Guariso G. et al. presented a general approach to the System Assessment Model (IAM), namely, the driving force, pressure, state, impact, and response (DPSIR) scheme combined with specific cases of local air quality policies [14]. The method has been widely used in European countries. Liang C.S. et al. developed a method for comparing the concentration of pollutants in the target and circulation areas (referred to as a circulation comparison method), and verified its effectiveness by conducting an air pollution assessment in the Beijing, Tianjin, Hebei, Yangtze River Delta, Pearl River Delta, and ChengduChongqing agglomeration in 2015 [15]. Wang Z.B. et al. adopted the Hierarchical Cross-regional Multi-directional Linkage (HCML) air pollution prevention and control model, and evaluated its air pollution control effect by moni- 
toring the levels of $\mathrm{SO}_{2}, \mathrm{NO}_{2}, \mathrm{PM}_{10}, \mathrm{PM}_{2.5}, \mathrm{O}_{3}, \mathrm{CO}$, and other pollutants from 112 monitoring points in 13 cities from 2014 to 2015 [16]. Wang Y. et al. proposed an improved inter-regional and inter-sectoral emission flow model. According to the model, the transmission mode of atmospheric pollutants $\left(\mathrm{SO}_{2}\right.$, soot, dust, and NOR) in the Beijing-Tianjin-Hebei urban agglomeration was analyzed [17]. Zhao X. et al. used the 2010-2015 air quality daily report of the Urumqi urban agglomeration to introduce a time series VAR model and study the interaction and contribution of air pollution among different cities [18]. Tanzarella A. et al. adopted the DELTA software tool to evaluate the air quality in the Apulia region of southern Italy in 2013 to identify sources of pollutants that primarily affect the air quality and implemented appropriate emission control strategies [19]. D'Elia I. et al. used the comprehensive evaluation model, MINNI, to evaluate the existing measures for the first time, which can also dynamically evaluate the pollution emissions [20]. Rao M. et al. studied the effect of the $\mathrm{PM}_{2.5}$ concentration on the Italian air quality by applying the MINNI model based on two sets of emission data. The study revealed the importance of two parameters, namely, the emission factors and uncertainty analysis of technology shares [21]. Sun Y. et al. analyzed the cooperation mechanism of smog governance linkage cooperation in the urban agglomeration of Liaoning Economic Zone, and established a comprehensive evaluation model of smog linkage collaboration, which can be used to supervise and evaluate the smog linkage coordination mechanism [22].

According to a literature review, through existing studies on urban or urban agglomeration air quality assessment, a wealth of results have been accumulated, namely, by adopting diverse application methods, combining qualitative and quantitative means, conducting theoretical and empirical research, and applying multidisciplinary studies. However, there remains issues to be further discussed related to an air quality assessment of urban agglomeration, which are described below.

First, it is of significant importance to aggregate the air pollution indices of each major city in an urban agglomeration to form an optimal aggregation of information and carry out a dynamic and comprehensive evaluation of the air quality of an entire urban agglomeration for different years. However, widely used methods, such as an averaging method, was used to determine the information set, the disadvantage of which is the fact that the original information cannot be fully utilized, which means the method lacks aggregation accuracy and will therefore cause deviations in the overall air quality assessment of urban agglomeration.

Second, it is difficult to reflect the overall air pollution in a city air pollution assessment system with a single index, and therefore the system is usually composed of multiple indices. The pollution indices of various cities in an urban agglomeration can be mapped into multi-dimensional spatial point sets, which will undoubtedly bring about difficulties regarding the solution of air pollution in- 
formation aggregation points.

In view of the above problems, in this paper, a comprehensive air quality evaluation method for urban agglomerations based on spatial Steiner-Weber points is proposed. By using this method, the air pollution information of each city can be aggregated into a set of spatial points. A PGSA was then applied to solve the optimal aggregation points, which have no loss of information (deviating from the optimal points will bring about information loss). For the calculation of indices with attribute weights, instead of using subjective weighting methods such as AHP and Delphi, in this study, a coefficient of variation method is applied that can reflect the importance of the indices to determine the indices' weights. Finally, we took the Beijing-Tianjin-Hebei urban agglomeration as an empirical research object, and the air quality of each year was dynamically evaluated and compared based on data from the China Statistical Yearbook. The purpose of this paper is to develop a new theoretical method to solve the problem of evaluating air quality of the entire urban agglomeration through isolated local air quality information of different cities. This paper also provides a reference basis to further dig out mechanism among cities in one urban agglomeration and between different urban agglomerations.

\section{Preliminary Knowledge}

\subsection{Prototype Model: Steiner-Weber Point Problem}

\subsubsection{Plane Steiner-Weber Point Problems}

The Steiner-Weber point problem took its shape from the Fermat-Torricelli point problem, which was first proposed by the French mathematician Fermat in 1643. Torricelli was later the first to solve the problem by using elementary geometry, and therefore, this point has often been referred to as the Fermat-Torricelli point. The Fermat-Torricelli point problem is a suitable tool for locating the point with the smallest sum of the three vertices from any planar triangle [23]. Since then, Steiner and Weber have substantially expanded the Fermat-Torricelli point, which is also called the Steiner-Weber point. When applying SteinerWeber points under the condition in which $n$ known points on the plane (where $n$ can be bigger than 3) are given, the point that satisfies the shortest sum of weighted distances to these $\mathrm{n}$ known points is solved [24], the mathematical model of which is as follows:

$$
\min _{x} f(x)=\sum_{i=1}^{m} \lambda_{i}\left\|x-a_{i}\right\|
$$

where the vectors $a_{1}, a_{2}, \cdots, a_{m} \in R^{n} ; m \geq 3$; the weights $\lambda_{i}>0$; and $\|\cdot\|$ is the Euclid vector norm.

Theorem $1 f(x)$ does not have to be differentiable everywhere, but must be convex.

Theorem 2 If there is no collinearity among $a_{1}, a_{2}, \cdots, a_{m}, f(x)$ must have a unique solution. 


\subsubsection{Steiner-Weber Spatial Point Problem}

Planar Steiner-Weber point problems can also be extended to spatial Steiner-Weber point problems. Its mathematical model is as follows:

Let the point set of $\mathrm{m}$ known points in Euclidian space $R^{n}$ be the following:

$$
\left\{A^{i}\left(x_{1}^{i}, x_{2}^{i}, \cdots, x_{n}^{i}\right), i=1,2, \cdots, m\right\}
$$

If there is one point, $B^{*}\left(x_{1}^{*}, x_{2}^{*}, \cdots, x_{n}^{*}\right)$ is satisfied through the following:

$$
\min \lambda_{i}\left|A^{i} B^{*}\right|=\min \sum_{i=1}^{m} \lambda_{i}\left(\sum_{j=1}^{n}\left(x_{j}^{*}-x_{j}^{i}\right)^{2}\right)^{\frac{1}{2}}
$$

When weight $w_{i}>0, B^{*}$ can be called the Steiner-Weber point of $m$ known spatial points sets.

Intuitively speaking, let $n=3$ (the spatial coordinate system constituted by three-dimensional attributes) and $B^{* k}$ be the optimal aggregation point of a three-dimensional space, which is also known as Steiner-Weber point in three dimensions, as shown in Figure 1.

\subsection{Optimal Information Aggregation of Major Air Pollution Indices in Urban Agglomerations}

The following matrix can be used to represent the air pollution information of $m$ cities, during $p$ years and $n$ attributes, of different urban agglomerations:

$$
\begin{aligned}
A_{1} & =\left(\begin{array}{cccc}
x_{11}^{1} & x_{12}^{1} & \cdots & x_{1 n}^{1} \\
x_{21}^{1} & x_{22}^{1} & \cdots & x_{2 n}^{1} \\
\vdots & \vdots & \ddots & \vdots \\
x_{m 1}^{1} & x_{m 2}^{1} & \cdots & x_{m n}^{1}
\end{array}\right) \\
A_{2} & =\left(\begin{array}{cccc}
x_{11}^{2} & x_{12}^{2} & \cdots & x_{1 n}^{2} \\
x_{21}^{2} & x_{22}^{2} & \cdots & x_{2 n}^{2} \\
\vdots & \vdots & \ddots & \vdots \\
x_{m 1}^{2} & x_{m 2}^{2} & \cdots & x_{m n}^{2}
\end{array}\right)
\end{aligned}
$$

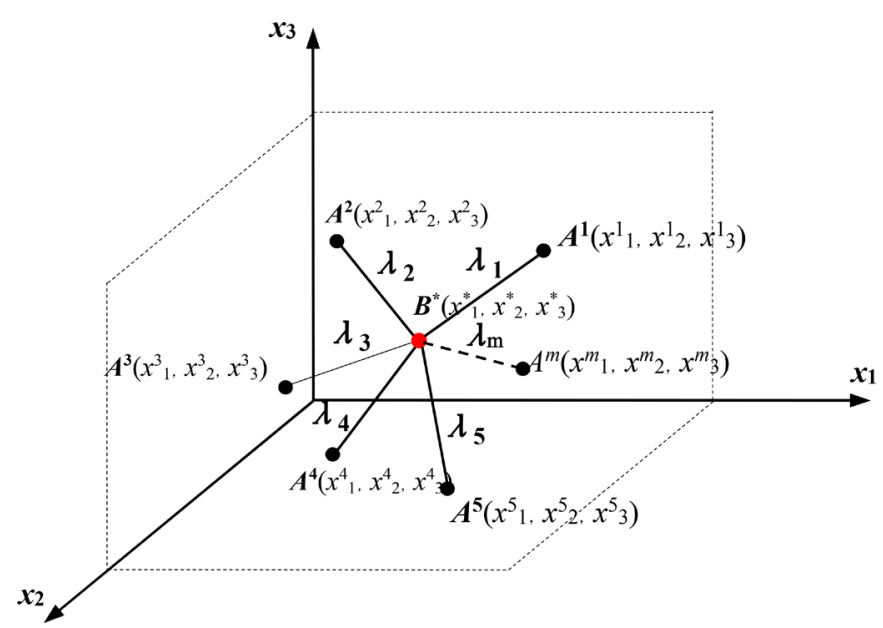

Figure 1. Steiner-Weber point diagram of three-dimensional space. 


$$
A_{P}=\left(\begin{array}{cccc}
x_{11}^{P} & x_{12}^{P} & \cdots & x_{1 n}^{P} \\
x_{21}^{P} & x_{22}^{P} & \cdots & x_{2 n}^{P} \\
\vdots & \vdots & \ddots & \vdots \\
x_{m 1}^{P} & x_{m 2}^{P} & \cdots & x_{m n}^{P}
\end{array}\right)
$$

where $x_{j i}^{k}$ is the $k$ th year $(k=1,2, \cdots, p)$ and the $j$ th city $(j=1,2, \cdots, m)$ to the th monitoring index value $(i=1,2, \cdots, n)$. Matrices $A_{1}, A_{2}, \cdots, A_{p}$ are mapped to $p$ n-dimensional Euclid spaces, $E^{n_{1}}, E^{n_{2}}, \cdots$ and $E^{n_{p}}$.

Definition 1. Let Euclid spaces be $E^{n_{1}}, E^{n_{2}}, \cdots, E^{n_{p}}$, when the following conditions are met:

$$
\bar{x}_{j i}^{k}=\frac{1}{m} \sum_{j=1}^{m}\left(x_{j i}^{k}\right)
$$

where $\bar{x}_{j i}^{k}$ is the $k$ th year $(k=1,2, \cdots, p)$ and th city $(j=1,2, \cdots, m)$ to the $i$ th monitoring index average value $(i=1,2, \cdots, n)$. Then, let $\bar{B}^{k}$ be the aggregation point of the mean value, and $\bar{B}=\left(\bar{B}^{1}, \bar{B}^{2}, \cdots, \bar{B}^{p}\right)$ be the mean aggregation point set. Therefore, the mean aggregation point set matrix can be presented as follows:

$$
\bar{R}=\left(\begin{array}{cccc}
\bar{x}_{1}^{1} & \bar{x}_{2}^{1} & \ldots & \bar{x}_{n}^{1} \\
\bar{x}_{1}^{2} & \bar{x}_{2}^{2} & \ldots & \bar{x}_{n}^{2} \\
\vdots & \vdots & \ddots & \vdots \\
\bar{x}_{1}^{p} & \bar{x}_{2}^{p} & \ldots & \bar{x}_{n}^{p}
\end{array}\right)
$$

Definition 2. Let the points set in Euclid spaces $E^{n_{1}}, E^{n_{2}}, \cdots, E^{n_{p}}$ be

$$
B_{j}^{k}=\left(x_{j 1}^{k}, x_{j 2}^{k}, \cdots, x_{j n}^{k}\right), j=1,2, \cdots, m ; k=1,2, \cdots, p
$$

under the condition

$$
d_{k}=\min \sum_{j=1}^{m}\left\|B_{j}^{k} B^{* k}\right\|=\min \sum_{j=1}^{m}\left(\sum_{i=1}^{n}\left(x_{j i}^{k}-x_{i}^{* k}\right)\right)^{\frac{1}{2}} \quad(k=1,2, \cdots, p)
$$

When case $B^{* k}$ is an optimal aggregation point $(k=1,2, \cdots, p)$, $B^{*}=\left(B^{* 1}, B^{* 2}, \cdots, B^{* p}\right)$ is an optimal aggregation point set.

In solving formula (4), the optimal aggregation point set matrix can be obtained as follows:

$$
R^{*}=\left(\begin{array}{cccc}
x_{1}^{* 1} & x_{2}^{* 1} & \cdots & x_{n}^{* 1} \\
x_{1}^{* 2} & x_{2}^{* 2} & \cdots & x_{n}^{* 2} \\
\vdots & \vdots & \ddots & \vdots \\
x_{1}^{* p} & x_{2}^{* p} & \cdots & x_{n}^{* p}
\end{array}\right)
$$

Solving formula (4) is equivalent to solving point set $B^{*}$ with the shortest Euclidean distance to $m$ known points in $p n$-dimensional Euclid spaces. When $n=$ 2 and $m=3$, it can be equivalent to the Fermat-Torricelli point problem, which can be solved using elementary geometry. When $n=2$ and $m>3$, it is equivalent to solving the planar Steiner-Weber point problem, which is usually solved 
through the centroid method and the differential partial derivative method. In addition, when $n>2$ and $m>3$, the spatial point problem is solved. At present, there are few references to algorithms for solving this problem in related studies. Thus, in this study, a biomimetic algorithm to simulate the growth of plants in nature, namely, the PGSA algorithm is used.

\subsection{Algorithm for Solving the Problem of Spatial Optimal Aggregation Points}

The plant growth simulation algorithm (PGSA) is an intelligent optimization algorithm using the plant-to-light mechanism as a heuristic criterion [25], which was proposed by the Tong in 2005. This algorithm takes the solution space of the optimization problem as the growth environment of the plant, and uses the optimal solution as the light source. According to the photo-light characteristics of real plants, the deductive mode of the branches and leaves growing rapidly toward the sunlight under different light intensity environments. Figure 2 shows a schematic diagram simulating the photo-growth of plants. The PGSA has attracted the attention of numerous scholars both at home and abroad, who have applied it to respective research fields, the results of which have turned out to be much better than those of other intelligent algorithms [26]-[38].

The implementation steps of the PGSA are as follows:

Step 1. Determine the initial growth point $x^{0} \in X$ and the step size $l / 1000$, in which $l$ is the length of the bounded closed box. Let $X_{\min }=x^{0}$, $F_{\min }=f\left(x^{0}\right)$, in which $f\left(x^{0}\right)$ is the backlight function of $x^{0}$.

Step 2. Let $x^{0}$ be the center point, and draw a line parallel to the $x$-axis and another parallel to the $y$-axis along a two-dimensional plane; $a_{1} \leq x_{1}^{0} \leq b_{1}, a_{2} \leq x_{2}^{0} \leq b_{2}, \cdots, a_{t} \leq x_{t}^{0} \leq b_{t}$ is then derived as new branches of growth. Look for $S_{i_{1} j_{1}}^{0}\left(1 \leq i_{1} \leq t, 1 \leq j_{1} \leq k_{1}\right)$ in $E$, in which $S_{i_{1} j_{1}}^{0}$ is the $j_{1}$ th growth point on the $i_{1}$ th branch.

Step 3. Compare $f\left(S_{i_{1} j_{1}}^{0}\right)$ with $F_{\min }$, if $f\left(S_{i_{1} j_{1}}^{0}\right) \leq F_{\min }$, then $X_{\min }=S_{i_{1} j_{1}}^{0}, F_{\text {min }}=f\left(S_{i_{1} j_{1}}^{0}\right)$. Otherwise, keep $X_{\text {min }}$ and $F_{\text {min }}$ unchanged.

Step 4. If $f\left(x^{0}\right) \leq f\left(S_{i_{1} j_{1}}^{0}\right)$, then its auxin concentration is $C_{S_{i_{j} j_{1}}^{0}}=0$; otherwise, solve $C_{s_{i_{1} j_{1}}^{0}}$ as follows:

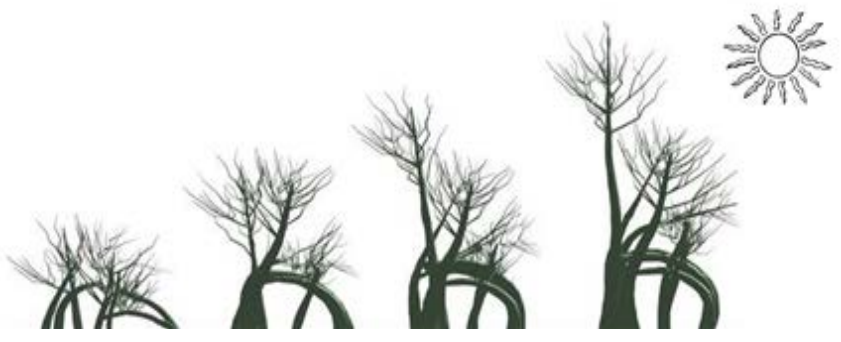

Figure 2. Schematic diagram of phototropism simulation of plant growth. 


$$
C_{S_{i_{1} j_{1}}^{0}}=\frac{f\left(x^{0}\right)-f\left(S_{i_{1} j_{1}}^{0}\right)}{\sum_{i_{1}=1}^{m} \sum_{j_{1}=1}^{k_{1}}\left[f\left(x^{0}\right)-f\left(S_{i_{1} j_{1}}^{0}\right)\right]}
$$

Step 5. Using the concentration of auxin for all growth points to establish a concentration point between 0 and 1 , let $\zeta_{0}$ be a random point for the interval number, and thus $\sum_{i_{1}=1}^{r_{1}} \sum_{j_{1}=1}^{t_{1}-1} C_{S_{i_{1} j_{1}}^{0}}<\zeta_{0}<\sum_{i_{1}=1}^{r_{1}} \sum_{j_{1}=1}^{t_{1}} C_{S_{i_{1} j_{1}}^{0}}$. Next, choose $S_{r_{1} t_{1}}^{0}$ as the new growth point; meanwhile, $x^{1}=S_{r_{1} t_{1}}^{0}, X_{\min }=x^{1}, F_{\min }=f\left(x^{1}\right)$.

Step 6. Let $x^{1}$ be the ideal point, and draw a line parallel to the $x$-axis and another parallel to the $y$-axis along a two-dimensional plane; $S_{i_{1} j_{1}}^{1}\left(1 \leq i_{2} \leq t, 1 \leq j_{2} \leq k_{1}\right)$ is then derived as a new branch of growth. Afterward, search $S_{i_{1} j_{1}}^{1}\left(1 \leq i_{2} \leq t, 1 \leq j_{2} \leq k_{1}\right)$ in $E$.

Step 7. Comparing $f\left(S_{i_{2} j_{2}}^{0}\right)$ with $F_{\text {min }}$, if $f\left(S_{i_{2} j_{2}}^{0}\right) \leq F_{\text {min }}$, then $X_{\text {min }}=S_{i_{2} j_{2}}^{0}, F_{\text {min }}=f\left(S_{i_{2} j_{2}}^{0}\right)$; otherwise, keep $X_{\text {min }}$ and $F_{\text {min }}$ unchanged.

Step 8. Solve $C_{S_{i_{1} j_{1}}^{0}}$ and $C_{S_{i_{1} j_{2}}^{1}}$, and if $f\left(x^{0}\right) \leq f\left(S_{i_{1} j_{1}}^{1}\right)$, then its auxin concentration is $C_{S_{i_{1 j 1}}^{0}}=0$; otherwise, solve $C_{S_{i_{1} j}^{0}}$ as follows:

$$
\begin{aligned}
& C_{S_{i_{1} j_{1}}}=\frac{f\left(x^{0}\right)-f\left(S_{i_{1} j_{1}}^{0}\right)}{\sum_{i_{1}=1}^{m} \sum_{j_{1}=1}^{k_{1}}\left[f\left(x^{0}\right)-f\left(S_{i_{1} j_{1}}^{0}\right)\right]+\sum_{i_{1}=1}^{m} \sum_{j_{1}=1}^{k_{2}}\left[f\left(x^{0}\right)-f\left(S_{i_{2} j_{2}}^{1}\right)\right]}, \\
& C_{S_{i_{2} j_{2}}^{1}}=\frac{f\left(x^{0}\right)-f\left(S_{i_{2} j_{2}}^{1}\right)}{\sum_{i_{1}=1}^{m} \sum_{j_{1}=1}^{k_{1}}\left[f\left(x^{0}\right)-f\left(S_{i_{1} j_{1}}^{0}\right)\right]+\sum_{i_{1}=1}^{m} \sum_{j_{1}=1}^{k_{2}}\left[f\left(x^{0}\right)-f\left(S_{i_{2} j_{2}}^{1}\right)\right]}
\end{aligned}
$$

Step 9. Using the concentration of auxin for all growth points to establish a concentration point between 0 and 1 , let $\zeta_{1}$ be another random point for the interval number, and thus $\sum_{i_{1}=1}^{r_{2}} \sum_{j_{1}=1}^{t_{2}-1} C_{S_{i_{1} j_{1}}^{0}}<\zeta_{1} \leq \sum_{i_{1}=1}^{r_{2}} \sum_{j_{1}=1}^{t_{2}} C_{S_{i_{1} j_{1}}^{0}}$. Next, choose $S_{r_{1} t_{1}}^{0}$ as the new growth point, and meanwhile $x^{2}=S_{r_{1} t_{1}}^{0}, X_{\min }=x^{2}, F_{\min }=f\left(x^{2}\right)$; otherwise, $\sum_{i_{1}=1}^{m} \sum_{j_{1}=1}^{k_{1}} C_{S_{i_{j} j_{1}}^{0}}+\sum_{i_{1}=1}^{r_{2}} \sum_{j_{1}=1}^{t_{2}} C_{S_{i_{2} j_{2}}^{1}}<\zeta_{1} \leq \sum_{i_{1}=1}^{m} \sum_{j_{1}=1}^{k_{1}} C_{S_{i_{j} j_{1}}^{0}}+\sum_{i_{1}=1}^{r_{2}} \sum_{j_{1}=1}^{t_{2}} C_{S_{i_{2} j_{2}}^{1}}$, and then choose $S_{r_{2} t_{2}}^{1}$ as the new growth point, through which we suppose $x^{2}=S_{r_{1} t_{1}}^{1}, X_{\min }=x^{2}, F_{\min }=f\left(x^{2}\right)$.

Step 10. Repeat steps 6 through 9 until $F_{\min }$ remains unchanged, and then determine whether $x^{*}=X_{\min }$ is the global optimal solution, and if so, the iterations end.

\subsection{Determine Attribute Weights and Assess the Schemes}

In this paper, the coefficient of variation method, the objective weighting method of the information contained in each index attribute, is applied to deter- 
mine the weights of the indices attributes. The specific steps are as follows::

- Calculate the mean $\bar{X}_{i}$ and standard deviation $\sigma_{i}$ of each index sample as follows:

$$
\begin{gathered}
\bar{X}_{i}=\frac{1}{n} \sum_{i=1}^{n} X_{i} \\
\sigma_{i}=\sqrt{\frac{1}{n} \sum_{i=1}^{n}\left(X_{i}-\bar{X}_{i}\right)^{2}}
\end{gathered}
$$

- Calculate the coefficient of variation $v_{i}$ and weight of each index $w_{i}$

The calculation formula for the coefficient of variation of each index is as follows:

$$
\begin{gathered}
v_{i}=\frac{\sigma_{i}}{\bar{X}_{i}} \\
w_{i}=\frac{v_{i}}{\sum_{i=1}^{n} v_{i}}
\end{gathered}
$$

- Evaluation of the scheme

Apply linear weighting method to evaluate the following scheme:

$$
\varphi(A)=W \cdot\left(R^{*}\right)^{\mathrm{T}}
$$

In this formula, $A=\left(A_{1}, A_{2}, \cdots, A_{p}\right), W=\left(w_{1}, w_{2}, \cdots, w_{n}\right)$, and $\varphi(A)$ is the comprehensive evaluation value of $p$ years.

\section{Empirical Research}

Take the agglomeration of Beijing-Tianjin-Hebei as the research object, and evaluate the air quality of the urban agglomeration dynamically from 2014 to 2017.

\subsection{Data Standardization}

To make the index data dimensionless, the raw data [39] can be processed through cost-based extreme value processing.

$$
X_{i j}=\frac{x_{j}^{\max }-x_{i j}}{x_{j}^{\max }-x_{j}^{\min }}(i=1,2, \cdots, n ; j=1,2, \cdots, m)
$$

The processed index data are shown in Table 1.

\subsection{Solution to Optimal Aggregation Points}

First, the PGSA solution is used to solve Formula (6), and the air pollution information aggregation points of the air pollution of $\mathrm{SO}_{2}, \mathrm{NO}_{2}$, and $\mathrm{PM}_{2.5}$ in years $A_{1}, A_{2}, A_{3}$, and $A_{4}$ (Figures 3-6) in the major cities of the Beijing, Tianjin, and Hebei agglomeration are obtained and applied to the optimal aggregation matrix in (16). To compare the method with the traditional means of averaging, the mean aggregation matrix in (17) is obtained by Equation (4). 


$$
R^{*}=\left(\begin{array}{lll}
0.3180 & 0.5756 & 0.3400 \\
0.2690 & 0.5718 & 0.2656 \\
0.1910 & 0.2915 & 0.3293 \\
0.3503 & 0.5899 & 0.2760
\end{array}\right)
$$

Table 1. Index values after standardization of air quality in major cities in Beijing, Tianjin, and Hebei agglomeration.

\begin{tabular}{|c|c|c|c|c|c|c|c|c|c|c|c|c|}
\hline & \multicolumn{3}{|c|}{$\left(A_{1}\right) 2014$} & \multicolumn{3}{|c|}{$\left(A_{2}\right) 2015$} & \multicolumn{3}{|c|}{$\left(A_{3}\right) 2016$} & \multicolumn{3}{|c|}{$\left(A_{4}\right) 2017$} \\
\hline & $\mathrm{SO}_{2}$ & $\mathrm{NO}_{2}$ & $\mathrm{PM}_{2.5}$ & $\mathrm{SO}_{2}$ & $\mathrm{NO}_{2}$ & $\mathrm{PM}_{2.5}$ & $\mathrm{SO}_{2}$ & $\mathrm{NO}_{2}$ & $\mathrm{PM}_{2.5}$ & $\mathrm{SO}_{2}$ & $\mathrm{NO}_{2}$ & $\mathrm{PM}_{2.5}$ \\
\hline Beijing & 1.0000 & 0.2727 & 0.6324 & 1.0000 & 0.5789 & 0.2989 & 1.0000 & 1.0000 & 0.4906 & 1.000 & 1.0000 & 0.6667 \\
\hline Tianjin & 0.4706 & 0.5455 & 0.6765 & 0.6341 & 1.0000 & 1.0000 & 0.6944 & 1.0000 & 0.5660 & 0.7500 & 0.6923 & 0.5714 \\
\hline Shijia-zhuang & 0.2157 & 0.6364 & 0.0735 & 0.1951 & 0.5263 & 0.2069 & 0.1389 & 0.0000 & 0.0000 & 0.2188 & 0.3846 & 0.0000 \\
\hline Tangshan & 0.0000 & 0.0000 & 0.4118 & 0.1463 & 0.0000 & 0.2529 & 0.0000 & 0.0000 & 0.4717 & 0.0000 & 0.0000 & 0.4763 \\
\hline Qinhuang-dao & 0.3725 & 1.0000 & 1.0000 & 0.4146 & 0.8421 & 0.6782 & 0.5000 & 1.0000 & 1.0000 & 0.4375 & 0.7692 & 1.0000 \\
\hline Handan & 0.3137 & 0.8182 & 0.2059 & 0.2439 & 0.7368 & 0.1839 & 0.1111 & 0.3000 & 0.3208 & 0.0000 & 0.6154 & 0.0000 \\
\hline Baoding & 0.1176 & 0.4545 & 0.0000 & 0.0000 & 0.3684 & 0.0000 & 0.1944 & 0.0000 & 0.1132 & 0.3438 & 0.6923 & 0.0476 \\
\hline
\end{tabular}

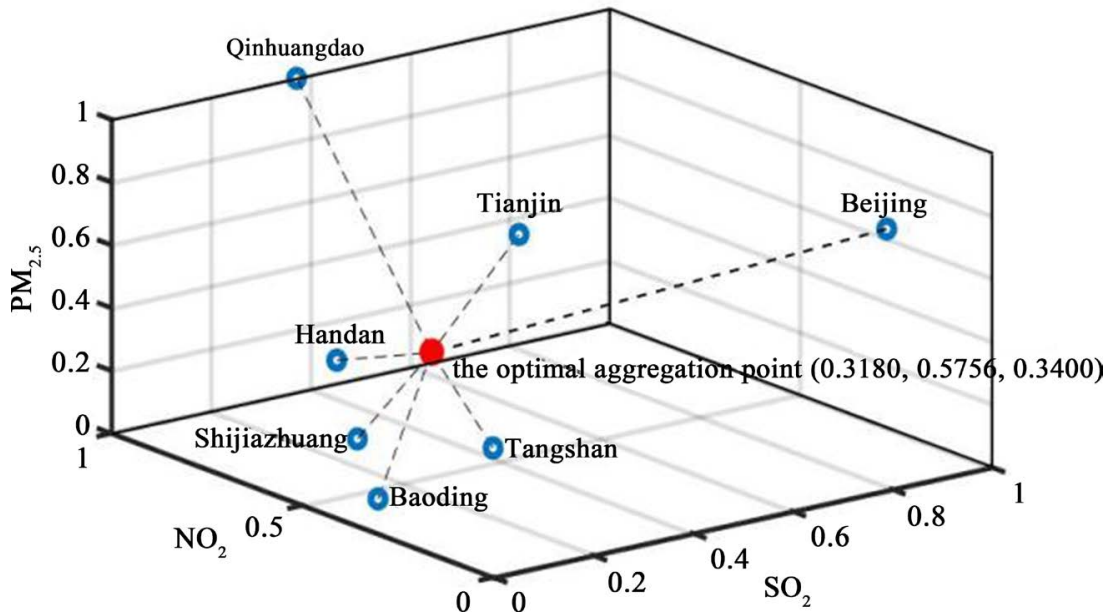

Figure 3. Aggregation in year $A_{1}$.

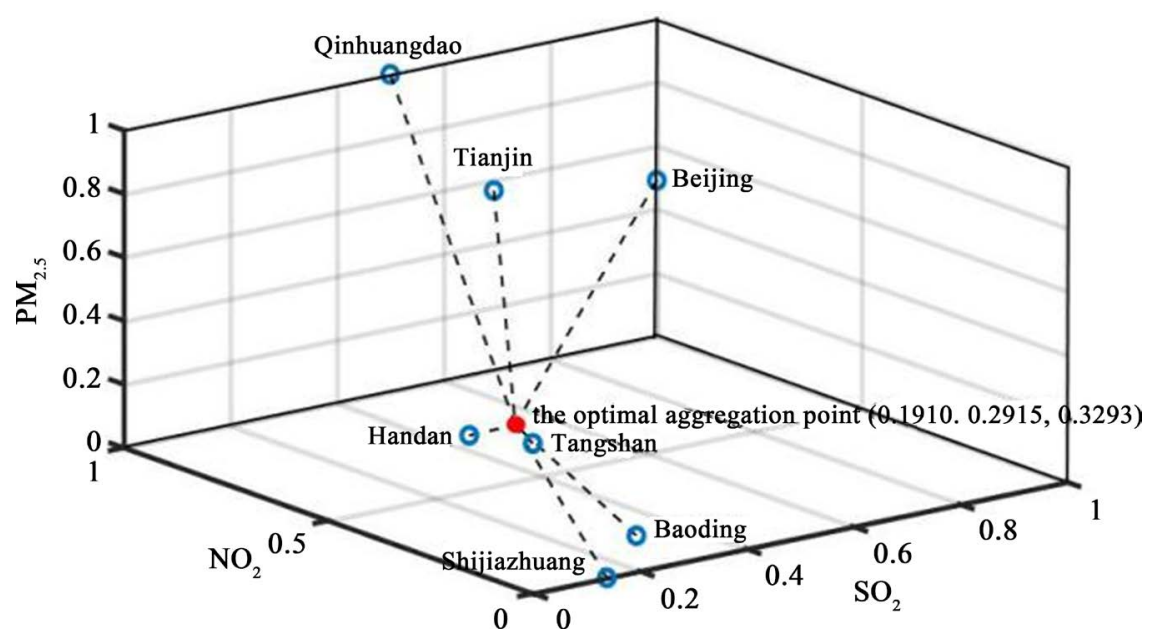

Figure 4. Aggregation in year $A_{2}$. 


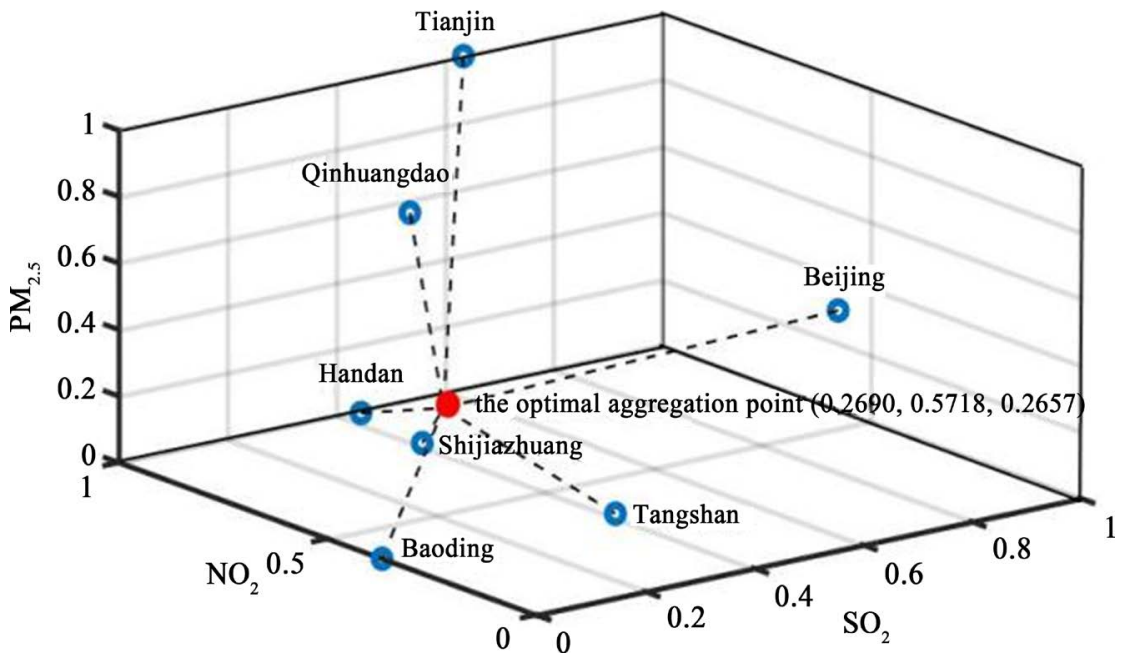

Figure 5. Aggregation in year $A_{3}$.

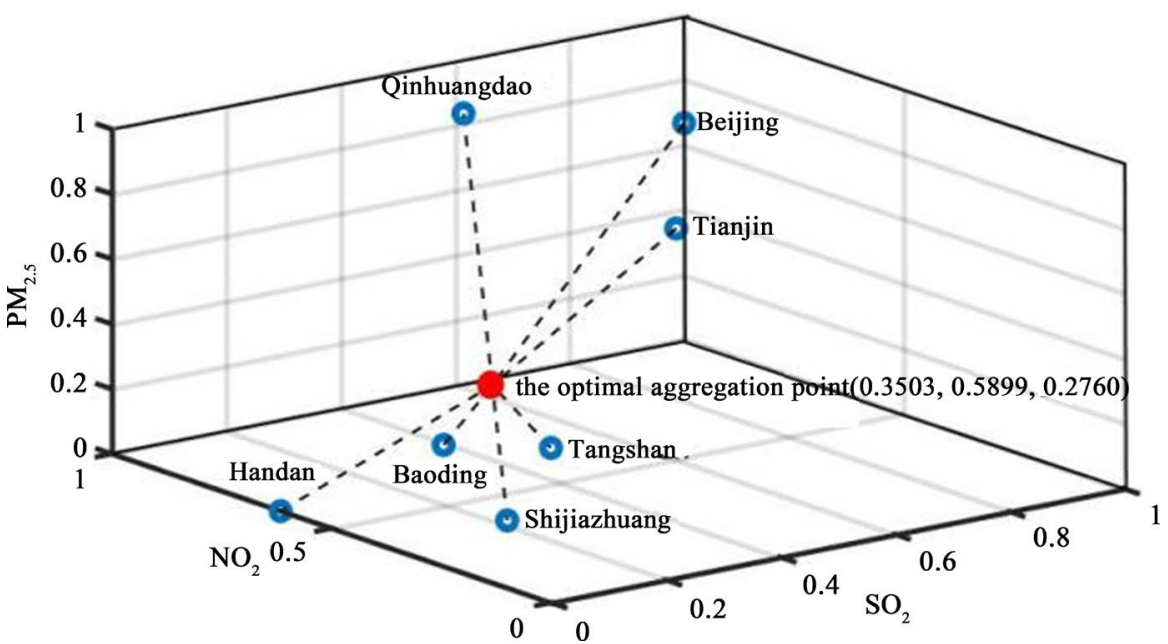

Figure 6. Aggregation in year $A_{4}$.

Table 2. Comparison of aggregation accuracy including two aggregation methods.

\begin{tabular}{ccc}
\hline & PGSA Method & Mean aggregation method \\
\hline$d_{1}\left(A_{1}\right)$ & 3.6022 & 3.6334 \\
$d_{2}\left(A_{1}\right)$ & 3.4761 & 3.5679 \\
$d_{3}\left(A_{1}\right)$ & 4.2746 & 4.4671 \\
$d_{4}\left(A_{1}\right)$ & 3.8088 & 3.9393 \\
\hline
\end{tabular}

$$
\bar{R}=\left(\begin{array}{lll}
0.3557 & 0.5325 & 0.4286 \\
0.3763 & 0.5789 & 0.3744 \\
0.3770 & 0.4714 & 0.4332 \\
0.3929 & 0.5934 & 0.3946
\end{array}\right)
$$

Let $d_{i}\left(A_{i}\right)$ be the sum of the distance from the $i$-year aggregation point to all known points $(i=1,2,3,4)$. Table 2 compares the aggregation accuracy of the two aggregation methods. The data in Table 2 indicate that the optimal aggrega- 
tion point solved using this method is of higher accuracy than the mean aggregation point (the smaller the sum of the aggregation points to all known points is, the higher the accuracy of the aggregation) of each year

\subsection{Calculation of Weights}

The weights of the index attributes are calculated according to Formula (11)(14), the results of which are shown in Table 3. It can be seen from Table 3 that the importance of each index attribute is $\mathrm{SO}_{2}, \mathrm{PM}_{2.5}, \mathrm{NO}_{2}$ in descending order.

\subsection{Calculation of Evaluation Value and Assessment}

According to Formula (15), the evaluation value $\varphi\left(A_{i}\right)$ is calculated as follows:

$$
\begin{aligned}
\varphi\left(A_{i}\right) & =(0.3657,0.2817,0.3526)\left[\begin{array}{lll}
0.3180 & 0.5756 & 0.3400 \\
0.2690 & 0.5718 & 0.2656 \\
0.1910 & 0.2915 & 0.3293 \\
0.3579 & 0.5922 & 0.2571
\end{array}\right]^{\mathrm{T}} \\
& =(0.3983,0.3531,0.2681,0.3884)^{\mathrm{T}}
\end{aligned}
$$

with normalized processing $\varphi^{\prime}\left(A_{i}\right)=(0.2829,0.2508,0.1904,0.2759)^{\mathrm{T}}$.

The evaluated values are derived as follows:

$$
A_{1}=0.2829, A_{2}=0.2508, A_{3}=0.1904 \text { and } A_{4}=0.2759
$$

Therefore, the final evaluation result is

$$
A_{3} \succ A_{2} \succ A_{4} \succ A_{1}
$$

This calculation result shows that air quality ranked in descending order from 2014 to 2017 in the Beijing-Tianjin-Hebei urban agglomeration is 2016, 2015, 2017, 2014.

Figure 7 shows a dynamic trend of the air quality of the Beijing-Tianjin-Hebei urban agglomeration from 2014 to 2017.

\section{Conclusions}

Targeting a comprehensive evaluation of the air quality in urban agglomerations, in this study, an optimal aggregation of air pollution indices in major cities of urban agglomerations was proposed, and the entire urban agglomeration was then dynamically evaluated using this new method.

Table 3. Calculation results based on attribute weights of each index.

\begin{tabular}{cccc}
\hline & $\mathrm{SO}_{2}$ & $\mathrm{NO}_{2}$ & $\mathrm{PM}_{2.5}$ \\
\hline $\bar{x}_{i}$ & 0.3796 & 0.5443 & 0.4054 \\
$\sigma_{i}$ & 0.3276 & 0.3619 & 0.3374 \\
$v_{i}$ & 0.8630 & 0.6649 & 0.8322 \\
$w_{i}$ & 0.3657 & 0.2817 & 0.3526
\end{tabular}




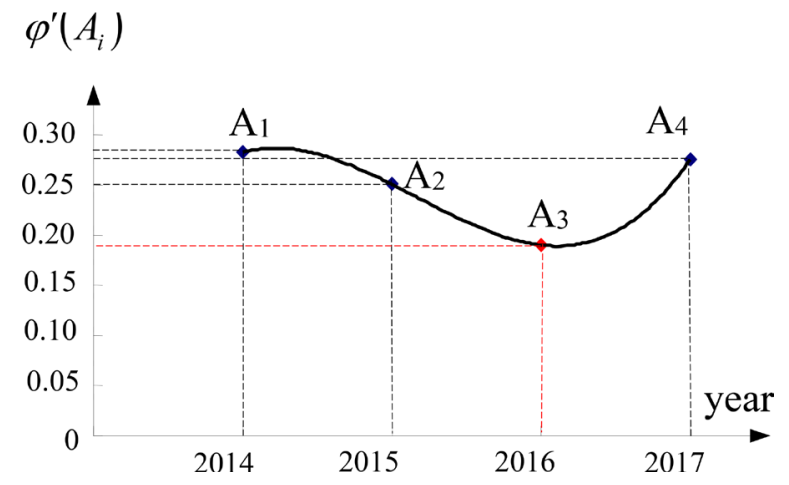

Figure 7. Dynamic trend of air quality of Beijing- Tianjin-Hebei urban agglomeration from 2014 to 2017.

Through this method, a main air pollution multi-index information matrix of an urban agglomeration was first mapped to a Euclidean space, and the $n$-dimensional ( $n$ indices) Euclidean space of $m$ cities in $p$ years was formed, and an optimal aggregation point formula was then established according to the minimum Euclidean spatial distance. Solving the optimal aggregation point is difficult (in fact, it is difficult to solve the aggregation point of $n$ known points even in a planar space) for an $n$-dimensional Euclidean space. Therefore, the simulated PGSA, which was developed in recent years and performs well in finding the global optimum, was used to optimize the aggregation points and obtain the aggregation matrix of the optimal aggregation points. In this paper, both modeling and optimization calculation processes were conducted using the PGSA, which means it yields a higher theoretical aggregation accuracy (some empirical studies have also strongly confirmed this) compared with the traditional mean method when solving the aggregation point. As for the calculation of the weights of the index attributes, in this study, subjective weighting methods such as AHP and Delphi have yet to be adopted, but the coefficient of variation method, which can reflect the importance among the indices used to determine the weights, has been used.

To indispensably apply and verify the method, in this paper, a dynamic empirical study of the urban agglomeration based on historical data of the air pollution indices of the major cities in the Beijing-Tianjin-Hebei agglomeration from the China Statistical Yearbook of 2014-2017 was conducted. It turned out that the change in air quality status of the Beijing-Tianjin-Hebei urban agglomeration has a certain volatility: 2016 was the best year, followed by 2015, 2017, and 2014 as the worst years considered. The results show that the air quality of urban agglomeration has not been fundamentally improved, and enterprises need to be further ameliorated through an industrial transformation and government supervision, thereby confirming the practicability and effectiveness of the method.

\section{Acknowledgements}

This work was supported in part by the Fund Program for Humanities and So- 
cial Sciences Research of the Ministry of Education under Grant 17YJAZH041 and National Natural Science Foundation of China under Grant No. 71671022.

\section{Conflicts of Interest}

The authors declare no conflicts of interest regarding the publication of this paper.

\section{References}

[1] Dong, X.L. (2012) Environmental Problem Observation of Urban Agglomerations and Environmental Model Exploration. Environmental Protection, No. 4, 42-44.

[2] Wang, H.H. and Lu, Z.H. (2017) The Impact of Economic Agglomeration on Urban Agglomeration of Urban Air Quality: A Case Study of the Yangtze River Delta Urban Agglomeration. China Market, No. 17, 295-298.

[3] Muezzinoglu, A., Atimtay, A.T. and Incecik, S. (2008) Special Issue: Assessment of Urban and Regional Air Quality and Its Impacts-Selected Papers Submitted to the Third International Symposium on Air Quality Management at Urban, Regional and Global Scales (AQM2005) and 14th IUAPPA (the International Union of Air Pollution Prevention and Environmental Protection Associations) Regional Conference Held in Istanbul on September 26-30, 2005-Preface. Environment International, 34, 579-579. https://doi.org/10.1016/j.envint.2007.12.003

[4] Yue, D.L., Xie, M., Zhou, Y., et al. (2013) Impact of New Environmental Air Quality Standards on Air Quality Assessment of the Pearl River Delta Regional Station. Environmental Monitoring in China, 29, 38-42.

[5] Zhou, S.D., Ouyang, W.Q. and Ge, J.H. (2017) Study on the Main Influencing Factors and Internal Relations between Them of $\mathrm{PM}_{2.5}$ in Beijing-Tianjin-Hebei Agglomeration. China Population Resources and Environment, 27, 102-109.

[6] Chen, K., Wang, Z.W., Liu, Z.H., et al. (2017) Time Evolution Characteristics of Air Pollution in Southern Sichuan Urban Agglomeration from 2005 to 2014. Environmental Engineering, 35, 72-76, 81.

[7] Liu, H.M., Fang, C.L., Huang, X.J., et al. (2018) Analysis of Temporal and Spatial Characteristics and Influencing Factors of Air Pollution in Beijing-Tianjin-Hebei Urban Agglomeration. Acta Geographica Sinica, 73, 177-191.

[8] Xion, Y. and Liu, Y. (2018) Research on Air Pollution Control Evaluation of Beijing-Tianjin-Hebei and Yangtze River Delta Urban Agglomerations-Based on PSR Evaluation System. China Development, 18, 13-18.

[9] Chen, S.Y. and Wang, J.M. (2018) Study on the Evaluation and Policy Path of Urban Haze Management in China: Taking the Yangtze River Delta as an Example. China Population Resources and Environment, 28, 71-80.

[10] Coelho, M.C., Fontes, T., Bandeira, J.M., et al. (2014) Assessment of Potential Improvements on Regional Air Quality Modelling Related with Implementation of a Detailed Methodology for Traffic Emission Estimation. Environment International, 470, 127-137. https://doi.org/10.1016/j.scitotenv.2013.09.042

[11] Wang, N., Lyu, X.P. and Deng, X.J. (2016) Assessment of Regional Air Quality Resulting from Emission Control in the Pearl River Delta Region, Southern China. Science of the Total Environment, 573, 1554-1565.

https://doi.org/10.1016/j.scitotenv.2016.09.013

[12] Thunis, P., Degraeuwe, B., Pisoni, E., et al. (2016) On the Design and Assessment of 
Regional Air Quality Plans: The SHERPA Approach. Journal of Environmental Management, 183, 952-958. https://doi.org/10.1016/j.jenvman.2016.09.049

[13] Thunis, P., Miranda, A., Baldasano, J.M., et al. (2016) Overview of Current Regional and Local Scale Air Quality Modelling Practices: Assessment and Planning Tools in the EU. Environmental Science \& Policy, 65, 13-21.

https://doi.org/10.1016/j.envsci.2016.03.013

[14] Guariso, G., Maione, M. and Volta, M. (2016) A Decision Framework for Integrated Assessment Modelling of Air Quality at Regional and Local Scale. Environmental Science \& Policy, 65, 3-12. https://doi.org/10.1016/j.envsci.2016.05.001

[15] Liang, C.S., Liu, H. and He, K.B. (2016) Assessment of Regional Air Quality by a Concentration-Dependent Pollution Permeation Index. Scientific Reports, 6, Article No. 34891. https://doi.org/10.1038/srep34891

[16] Wang, Z.B., Liang, L.W., Lin, X.B., et al. (2017) Control Models and Effect Evaluation of Air Pollution in Jing-Jin-Ji Urban Agglomeration. Environmental Science, 38, 4005-4014.

[17] Wang, Y., Liu, H.W., Mao, G.Z., et al. (2017) Inter-Regional and Section Linkage Analysis of Air Pollution in Beijing-Tianjin-Hebei (Jing-Jin-Ji) Urban Agglomeration of China. Journal of Cleaner Production, 165, 1436-1444. https://doi.org/10.1016/j.jclepro.2017.07.210

[18] Zhao, X.P., Zibibula, S.Y., Feng, G., et al. (2017) Analysis of Mutual Influence of Air Pollution among Cities in Urumqi Urban Agglomeration. Chinese Journal of Environmental Engineering, 35, 96-100, 64.

[19] Tanzarella, A., Schipa, I., Morabito, A., et al. (2017) Application of a Photochemical Model for the Assessment of Regional Air Quality in Southern Italy: Procedures and Results. Environmental Science, 62, 102-126. https://doi.org/10.1504/IJEP.2017.10010369

[20] D’Elia, I., Piersanti, A., Briganti, G., et al. (2018) Evaluation of Mitigation Measures for Air Quality in Italy in 2020 and 2031. Atmospheric Pollution Research, 9, 977988. https://doi.org/10.1016/j.apr.2018.03.002

[21] Rao, M., D’Elia, I. and Piersanti, A. (2018) An Uncertainty Quantification of PM2.5 Emissions from Residential Wood Combustion in Italy. Atmospheric Pollution Research, 9, 526-533. https://doi.org/10.1016/j.apr.2017.12.002

[22] Sun, Y.L., Liu, J., He, H.Y., et al. (2018) Research on Comprehensive Evaluation Indices System in Harmony Cooperation Mechanism of Urban Agglomeration in Liaoning Economic Zone. Journal of Shenyang Jianzhu University (Natural Science Edition), 34, 375-384.

[23] Kupitz, Y.S., Martini, H. and Spirova, M. (2013) The Fermat-Torricelli Problem, Part I: A Discrete Gradient-Method Approach. Journal of Optimization Theory and Applications, 158, 305-327. https://doi.org/10.1007/s10957-013-0266-Z

[24] Dietmar, C. (1988) The Fermat-Steiner-Weber-Problem in Minkowski Spaces. Optimization A Journal of Mathematical Programming and Operations Research, 19, 485-489. https://doi.org/10.1080/02331938808843367

[25] Tong, L., Wang, C.F., Su, W.L., et al. (2005) A Bionic Global Optimization Algorithm for Solving Integer Programming-Simulated Plant Growth Algorithm. System Engineering-Theory \& Practice, 25, 76-85.

[26] Tong, L. and Wang, Z.T. (2008) Application of Simulated Plant Growth Algorithm in Facility Location Problem. System Engineering-Theory \& Practice, 28, 107-115. https://doi.org/10.1016/S1874-8651(10)60025-7 
[27] Guney, K., Durmus, A. and Basbug, S. (2009) A Plant Growth Simulation Algorithm for Pattern Nulling of Linear Antenna Arrays by Amplitude Control. Progress in Electromagnetics Research B, 17, 69-84. https://doi.org/10.2528/PIERB09061709

[28] Rao, R.S. and Narasimham, S. (2009) A New Heuristic Approach for Optimal Network Reconfiguration in Distribution Systems. Journal of Applied Science, Engineering and Technology, 5, 15-21.

[29] Thiruvenkadam, S., Nirmalkumar, A. and Sakthivel, A. (2009) Optimal Reconfiguration Algorithm for Radial Distribution System Feasible under Normal and Abnormal Conditions. International Journal of Applied Engineering Research, 4, 115-122.

[30] Sirjani, R., Mohamed, A. and Shareef, H. (2010) Optimal Capacitor Placement in a Radial Distribution System Using Harmony Search Algorithm. Journal of Applied Sciences, 10, 2998-3006. https://doi.org/10.3923/jas.2010.2998.3006

[31] Sarma, A.K. and Rafi, K.M. (2011) Optimal Selection of Capacitors for Radial Distribution Systems Using Plant Growth Simulation Algorithm. International Journal of Advanced Science and Technology, 30, 43-54.

[32] Kumar, S.A. and Goud, K.J. (2012) Power Loss Reduction in Radial Distribution System by Using Plant Growth Simulation Algorithm. Journal of Energy Technologies \& Policy, 3, 14-25.

[33] Lu, S. and Yu, S.A. (2014) Fuzzy k-Coverage Approach for RFID Network Planning Using Plant Growth Simulation Algorithm. Journal of Network \& Computer Applications, 39, 280-291. https://doi.org/10.1016/j.jnca.2013.07.015

[34] Grebenyuk, G.G. and Krygin, A.A. (2015) Limit Graphs in Structural Optimization of Modes in Distribution Networks. Automation \& Remote Control, 76, 120-132. https://doi.org/10.1134/S0005117915010117

[35] Bhattacharjee, D., Paul, A., Kim, J.H., et al. (2016) An Object Localization Optimization Technique in Medical Images Using Plant Growth Simulation Algorithm. Date of Electronic Publication, 5, 1784. https://doi.org/10.1186/s40064-016-3444-2

[36] Wang, X.P., Qiu, J., Tong, L., et al. (2017) A Network Optimization Research for Product Returns Using Modified Plant Growth Simulation Algorithm. Scientific Programming, 9, 1-14. https://doi.org/10.1155/2017/1080468

[37] Ren, X.Y., Kong, Z.F., Liang, W.C., et al. (2017) Vehicle Scheduling Based on Plant Growth Simulation Algorithm and Distribution Staff Behavior. Advances in Production Engineering \& Management, 12, 173-184. https://doi.org/10.14743/apem2017.2.249

[38] Shi, K.R., Ruan, Z.J., Jiang, Z.R., et al. (2018) Improved Plant Growth Simulation and Genetic Hybrid Algorithm (PGSA-GA) and Its Structural Optimization. Engineering Computations, 35, 268-286. https://doi.org/10.1108/EC-03-2017-0113

[39] National Bureau of Statistics (2018) China Statistical Yearbook-2018. China Statistics Press, Beijing. 